\title{
Editorial: Age-Related Changes in Body Composition: Mechanisms, Clinical Implications and Possible Treatments
}

\author{
Anna Picca ${ }^{1 *}$, Francesco Landi ${ }^{1,2}$ and Matteo Cesari ${ }^{3,4}$ \\ ${ }^{1}$ Fondazione Policlinico Universitario "Agostino Gemelli" IRCCS, Rome, Italy, ${ }^{2}$ Institute of Internal Medicine and Geriatrics, \\ Università Cattolica del Sacro Cuore, Rome, Italy, ${ }^{3}$ Department of Clinical Sciences and Community Health, Università di \\ Milano, Milan, Italy, ${ }^{4}$ Geriatric Unit, Fondazione IRCCS Ca' Granda Ospedale Maggiore Policlinico, Milan, Italy
}

Keywords: aging, body composition, malnutrition, muscle aging, sarcopenia, diet, exercise, signaling pathways

\section{Editorial on the Research Topic}

Age-Related Changes in Body Composition: Mechanisms, Clinical Implications and Possible Treatments

Advancing age is characterized by remarkable changes in body composition that are not necessarily reflected by modifications in body weight (1). One notable body reshaping observed during aging is sarcopenia-the decline in muscle mass, function, and strength that increases the risk of incurring negative health-related outcomes (e.g., disability, loss of independence, institutionalization, death) (2). The loss of lean body mass is often masked by stable or even increased body weight as a consequence of greater adiposity, potentially resulting in the detrimental condition of sarcopenic obesity (3).

Sarcopenia is a "hot topic" in geriatrics. Nevertheless, to date, the condition is still orphan of

Edited and reviewed by: Tzvi Dwolatzky,

Technion Israel Institute of Technology, Israel

*Correspondence:

Anna Picca

anna.picca@guest.policlinicogemelli.it

Specialty section:

This article was submitted to

Geriatric Medicine,

a section of the journal

Frontiers in Medicine

Received: 05 March 2020 Accepted: 04 May 2020

Published: 14 July 2020

Citation:

Picca A, Landi F and Cesari M (2020) Editorial: Age-Related Changes in Body Composition: Mechanisms, Clinical Implications and Possible Treatments. Front. Med. 7:230 doi: $10.3389 /$ fmed.2020.00230 a univocal operational definition (2). The matter is further complicated by the heterogeneity of the clinical phenotypes, the complex pathophysiology, and the lack of standardized biomarkers. As frequently occurring with geriatric syndromes, the frequent superimposition of common conditions of advanced age complicates the study of a sarcopenia as a stand-alone disease (4). The ultimate consequences of such an impasse are the lack of incorporation of sarcopenia in everyday clinical practice and the absence of pharmacological treatments against the condition.

The research collection "Age-Related Changes in Body Composition: Mechanisms, Clinical Implications, and Possible Treatments" aimed at gathering contributions on age-related changes in body composition from different, yet complementary, points of view by convening clinicians and basic researchers working in the field of muscle aging in human and preclinical models.

In an up-to-date overview, Ziaaldini et al. illustrated the putative molecular mechanisms underlying the onset and progression of sarcopenia. These include biochemical pathways pertaining to insulin-like growth factor 1 (IGF-1)/Akt/mammalian target of rapamycin (mTOR), forkhead box $\mathrm{O}$ (FoxO) transcription factors, transforming growth factor (TGF)- $\beta$, nuclear factor $(\mathrm{NF})-\kappa \mathrm{B}$, and the mitogen-activated protein kinases (MAPKs). The effects of exercise on some of these signaling pathways are also discussed (Ziaaldini et al.).

Sarcopenia was also framed in the context of neuromuscular aging, focusing on dysfunctional neuromuscular junctions (NMJs) as a mechanism involved in muscle wasting by Casati et al. In this context, the dissection of brain-muscle cross talk during aging may help unveil novel biomarkers for the condition (Casati et al.). The synaptosomal-associated protein of $25 \mathrm{kDa}$ (SNAP25) was found to accumulate in the plasma membrane of nerve terminals at NMJs and 
regulate exocytosis of peripheral and central synapses in preclinical models (Casati et al.). Hence, SNAP25 may be a relevant pathway for understanding the molecular mechanisms regulating muscle homeostasis during aging, potentially supporting the clustering of biological determinants in sarcopenia.

Malnutrition, in particular, disarrangements in proteinamino acid metabolism, is a geriatric giant contributing to poor muscle function in older adults, especially in frail older patients $(5,6)$. As such, protein malnutrition is an actively investigated mechanism for the pathogenesis of sarcopenia. Nutritional interventions (as stand-alone interventions or in association with physical activity programs) are currently the most effective strategies for the management of sarcopenia (7). It is well-known that overall inadequate protein intake can impinge on muscle protein balance (i.e., affecting synthesis and degradation) and lead to skeletal muscle atrophy. However, only recently, it has become clear that protein quality matters as well. Indeed, the administration of diets with slightly imbalanced essential amino acids (EAA)/non-EAA blends to late middleaged mice induced body weight loss and tissue wasting, especially

\section{REFERENCES}

1. Roubenoff R. Sarcopenic Obesity: does muscle loss cause fat gain?: lessons from rheumatoid arthritis and osteoarthritis. Ann N Y Acad Sci. (2006) 904:553-7. doi: 10.1111/j.1749-6632.2000.tb06515.x

2. Landi F, Calvani R, Cesari M, Tosato M, Maria Martone A, Ortolani E, et al. Sarcopenia: an overview on current definitions, diagnosis and treatment. Curr Protein Pept Sci. (2018) 19:633-8. doi: 10.2174/13892037186661706071 13459

3. Marzetti E, Cesari M, Calvani R, Msihid J, Tosato M, Rodriguez-Mañas L, et al. The "Sarcopenia and Physical fRailty IN older people: multi-componenT Treatment strategies." (SPRINTT) randomized controlled trial: case finding, screening and characteristics of eligible participants. Exp Gerontol. (2018) 113:48-57. doi: 10.1016/j.exger.2018.09.017

4. Gingrich A, Volkert D, Kiesswetter E, Thomanek M, Bach S, Sieber CC, et al. Prevalence and overlap of sarcopenia, frailty, cachexia and malnutrition in older medical inpatients. BMC Geriatr. (2019) 19:120. doi: 10.1186/s12877-019-1115-1

5. Pasini E, Corsetti G, Aquilani R, Romano C, Picca A, Calvani R, et al. Protein-amino acid metabolism disarrangements: the hidden enemy of muscle atrophy (Corsetti et al.). Thus, an adequate EAA/nonEAA ratio is likely necessary for preserving whole-body energy status (Corsetti et al.).

Finally, the thickness of the quadriceps muscle assessed by bedside ultrasound was shown to predict rehospitalization and functional decline in older adults (Guerreiro et al.). Hence, this measure was indicated as a valuable tool for the evaluation of bedridden hospitalized older adults. In the same study, a contractile index was elaborated and associated with functional decline 3 months after hospital discharge (Guerreiro et al.).

Thanks to its multidisciplinary deployment, this collection provides an opportunity to increase awareness on the relevance of age-related changes in body composition and expand our knowledge on the pathophysiology of muscle aging.

\section{AUTHOR CONTRIBUTIONS}

All authors listed have made a substantial, direct and intellectual contribution to the work, and approved it for publication. chronic age-related conditions. Nutrients. (2018) 10:391. doi: 10.3390/nu100 40391

6. Landi F, Calvani R, Tosato M, Martone AM, Ortolani E, Savera G, et al. Protein intake and muscle health in old age: from biological plausibility to clinical evidence. Nutrients. (2016) 8:295. doi: $10.3390 /$ nu80 50295

7. Martone AM, Marzetti E, Calvani R, Picca A, Tosato M, Santoro L, et al. Exercise and protein intake: a synergistic approach against sarcopenia. Biomed Res Int. (2017) 2017:2672435. doi: 10.1155/2017/2672435

Conflict of Interest: The authors declare that the research was conducted in the absence of any commercial or financial relationships that could be construed as a potential conflict of interest.

Copyright (C) 2020 Picca, Landi and Cesari. This is an open-access article distributed under the terms of the Creative Commons Attribution License (CC BY). The use, distribution or reproduction in other forums is permitted, provided the original author(s) and the copyright owner(s) are credited and that the original publication in this journal is cited, in accordance with accepted academic practice. No use, distribution or reproduction is permitted which does not comply with these terms. 